\title{
Effects of signaling pathway inhibitors on hematopoietic stem cells
}

\author{
YUYU JIANG, ZHAOFENG XU, NA MA, LIZHI YIN, CAIQIN HAO and JING LI \\ Stem Cell Laboratory, Department of Biology, College of Life Sciences, \\ Shanghai Normal University, Shanghai 200234, P.R. China
}

Received October 25, 2019; Accepted August 11, 2020

DOI: $10.3892 / \mathrm{mmr} .2020 .11647$

\begin{abstract}
While there are numerous small molecule inhibitory drugs available for a wide range of signalling pathways, at present, they are generally not used in combination in clinical settings. Previous reports have reported that the effects of glycogen synthase kinase (GSK)3 $\beta$, p38MAPK, mTOR and histone deacetylase signaling combined together to suppress the stem-like nature of hematopoietic stem cells (HSCs), driving these cells to differentiate, cease proliferating and thereby impairing normal hematopoietic functionality. The present study aimed to determine the effect of HDACs, mTOR, GSK-3 $\beta$ and p38MAPK inhibitor combinations on the efficient expansion of HSCs using flow cytometry. Moreover, it specifically aimed to determine how inhibitors of the GSK3 $\beta$ signaling pathway, in combination with inhibitors of P38MAPK and mTOR signaling or histone deacetylase (HDAC) inhibitors, could affect HSC expansion, with the goal of identifying novel combination strategies useful for the expansion of HSCs. The results indicated that $\mathrm{p} 38 \mathrm{MAPK}$ and/or GSK3 $\beta$ inhibitors increased $\mathrm{Lin}^{-}$cell and $\mathrm{Lin}-\mathrm{Sca}-1^{+} \mathrm{c}-\mathrm{kit}^{+}(\mathrm{LSK})$ cell numbers in vitro. Taken together, these results suggested that a combination of p38MAPK and GSK $3 \beta$ signaling may regulate HSC differentiation in vitro. These findings further indicated that the suppression of p38MAPK and/or GSK $3 \beta$ signalling may modulate HSC differentiation and self-renewal to enhance HSC expansion.
\end{abstract}

\section{Introduction}

At present, the treatment of leukemia is dependent upon hematopoietic stem cell (HSC) transplantation (1), as HSCs are multipotent cells which can reconstitute the immune and hematological systems following irradiation (2-4). However, relatively few HSCs can be obtained from donor cord blood or bone marrow samples, and as such, the numbers obtained

Correspondence to: Dr Jing Li, Stem Cell Laboratory, Department of Biology, College of Life Sciences, Shanghai Normal University, 100 Guilin Road, Shanghai 200234, P.R. China

E-mail: 1ij@shnu.edu.cn

Key words: hematopoietic stem cells, glycogen synthase kinase $3 \beta$, p38MAPK, expansion in vitro are insufficient to meet clinical demands $(5,6)$. To date, numerous studies have reported that signaling through the p38MAPK, mTOR, glycogen synthase kinase (GSK) $3 \beta$ and histone deacetylases (HDACs) altered HSCs functionality in ways that were disadvantageous, impairing their ability to proliferate and driving the HSCs to undergo differentiation, thereby impairing the normal hematopoietic restoration of blood cells (7-9).

p38MAPK is an important MAPK, and the activation of p38MAPK signaling has been demonstrated to promote necrosis and differentiation, in addition to impairing the proliferation of HSCs $(7,10,11)$. It was previously reported that the inhibition of $\mathrm{p} 38 \mathrm{MAPK}$ rescued the regenerative defects of HSCs caused by reactive oxygen species production, prolonging the life of HSCs and maintaining their self-renewal (12). In addition, it was discovered that the inhibition of p38MAPK induced the homing of HSCs into the bone marrow in response to chemotactic factors (13), thereby improving the proliferation of leukemia cells or lymphocytes (14). Notably, p38MAPK signaling was also observed to be associated with chemotherapy resistance in patients with leukemia (15).

While first studied in the context of glycogen metabolism in the liver, GSK $3 \beta$ has also been identified as an important regulator of HSC homeostasis $(16,17)$. In a previous study, GSK3 $\beta$ activation was reported to drive HSC apoptosis and differentiation through p53 downregulation (18). Moreover, the inhibition of microRNA-126 regulated the PI3K/AKT/GSK3 $\beta$ signaling pathway and promoted the proliferation of HSCs (19). It was also illustrated that GSK $3 \beta$ promoted the migration of hematopoietic stem progenitor cells by regulating cytoskeletal rearrangement (20).

HDACs are able to induce histone lysine deacetylation, thereby modulating the progression of a wide range of diseases and biological processes (21). For example, the HDAC inhibitor valproic acid sodium salt (VPA) was discovered to enhance HSC proliferation, and as such, it is currently used in clinical contexts to treat myelodysplastic syndrome (22-25) and acute myeloid leukemia (AML) (26). Meanwhile, HDAC8 was discovered to aberrantly deacetylate p53 and promote leukemic stem cells(LSC) transformation and maintenance. Conversely, HDAC8 inhibition induced LSC apoptosis and restored p53 activity, suggesting the inhibition of HDAC8 as a promising approach to selectively target LSCs (27). mTOR signaling serves as a mechanism through which cells integrate inputs from numerous different factors, including oxygen, 
nutrients and cytokines, to induce appropriate growth, proliferation or survival responses (28).

At steady-state in vivo, the majority of HSCs remain dormant, with only a small fraction of progenitor cells serving to mediate blood cell production (29). However, under conditions of stress or inflammation, these HSCs can become activated and differentiate into additional progenitor cells, thereby enhancing the rate of blood cell replenishment (30). The rate of hematopoietic reconstitution in patients was discovered to be influenced by a wide range of variables, including the source of the donor HSCs, how many were transplanted and whether or not the recipient patients had undergone prior chemotherapy treatment (31). In a highly complex intracellular signaling network, the appropriate regulation of a signaling pathway is strongly dependent on the communication with other signaling molecules, resulting in synergistic or antagonistic relationships and a variety of biological effects (32). Thus, understanding the effects of inhibitors of different signaling pathways on the in vitro proliferation of HSCs is a promising strategy to promote the clinical application of HSCs.

Small molecule compounds that hold the potential to expand HSCs are of great promise in the stem cell transplantation field. Notably, current available small molecule compounds primarily affect several important signaling pathways, such as p38MAPK, mTOR, GSK3 $\beta$ and HDAC (31,33-35). Therefore, strategies to regulate these crucial signaling pathways may be of importance for effective HSC expansion in vitro. To effectively amplify available HSCs, the bone marrow microenvironment must be effectively mimicked in vitro without adversely impacting HSC activity (36). However, such mimicry is complex, as a wide range of mechanical and cytological stimuli work in concert in the bone marrow to modulate signaling pathway activation within these HSCs, thereby governing their ultimate functionality.

At present, research into expanding HSCs has predominantly focused on the following aspects: Promoting self-renewal, inhibiting differentiation, inhibiting apoptosis and promoting homing (13,37-39). HSCs are contained in the LSK cell population; phenotypically, LSK cells express stem cell antigen (Sca)-1 and c-Kit, but lack the lineage (Lin) markers expressed on mature myeloid and lymphoid cells (40). The present study aimed to investigate the efficacy of small molecule inhibitors on the manipulation of HSCs, especially the expansion of HSCs in vitro. Different combinations of inhibitors of the p38MAPK, mTOR, GSK $3 \beta$ and HDACs were used to treat HSC cultures at a range of concentrations, in order to explore which signaling pathways could be targeted to expansion HSCs more effectively in vitro. SB203580 was the first reported inhibitor of p38 $(41,42)$, which was discovered to permeate cells and inhibit the activation of MAPK activated protein kinase (MAPKAPK)-2 and MAPKAPK-3, which subsequently inhibits the partial signal transduction induced by certain inflammatory factors, such as IL-1 $\beta$ and TNF- $\alpha$. SB216763 is an effective, selective GSK3 $\beta$ inhibitor (43), which was discovered to activate glycogen synthase, stimulates glycogen synthesis in human hepatocytes, induces the expression of reporter genes regulated by $\beta$-catenin and promotes the accumulation of $\beta$-catenin, in which $\beta$-catenin is an important downstream effector of the Wnt signaling transduction pathway (44). Similarly, CHIR99021 is a GSK3 $\alpha$ and GSK3 $\beta$ inhibitor (45), which was illustrated to promote
HSC self-renewal, maintain colony morphology and regulate epigenetics by activating the Wnt signaling pathway $(46,47)$. The results of the present study demonstrated that suppressing p38MAPK and/or the GSK3 $\beta$ signaling pathway effectively amplified HSCs in vitro.

\section{Materials and methods}

Materials. The Cyan ADP flow cytometer, Moflo XDP flow cytometer and cryogenic high-speed centrifuge were all purchased from Beckman Coulter, Inc. The cell counter was obtained from Countstar. In addition, 10X Red Blood Cell Lysis buffer was acquired from eBioscience, $0.4 \%$ Trypan blue dye was provided by Sigma-Aldrich; Merck KGaA and FCS, SB203580, CHIR99021, rapamycin, VPA and SB216763 were purchased from Selleck Chemicals. The EasySeo ${ }^{\mathrm{TM}}$ Mouse SCA1 Positive Selection kit (cat. no. 18756) was provided by Stemcell Technologies, Inc.

Animal studies. Female C57BL6/J mice (age, 6-8 weeks, weight, 20-25g) were purchased from the Shanghai SLAC Laboratory Animal Co., Ltd. All mice were housed under a 12-h light/dark cycle in microisolator cages contained within a laminar flow ventilation system in the animal facility at the experimental Animal Center, Shanghai Normal University under specific-pathogen-free (SPF) conditions and used as donors to collect bone marrow HSCs. The mice were sacrificed by cervical dislocation ( $n=4$ per group). All animal studies were approved by the Institutional Animal Care and Use Committee of Shanghai Normal University.

Cell culture and flow cytometry. Bone marrow cells were isolated from murine femur and tibia bone marrow as previously described (48). Briefly, the bones were dissected and crushed three times with a pestle and, then the cells were collected in $6 \mathrm{ml}$ dissociation solution (PBS with 2\% FCS and $145 \mathrm{U} / \mathrm{ml}$ type-4 collagenase; Gibco; Thermo Fisher Scientific, Inc.) and incubated at $37^{\circ} \mathrm{C}$ for $30 \mathrm{~min}$. Samples were subsequently filtered using a 40- $\mu \mathrm{m}$ Nylon cell strainer to obtain single cell suspensions. The EasySep ${ }^{\mathrm{TM}}$ Mouse SCA1 Positive Selection kit was then used to isolate Sca1-PE positive cells.

Subsequently, the pre-prepared primary antibodies mixture (2\% FCS-PBS solution) containing anti-CD11b-biotin (clone no. M1/7; eBioscience; cat. no. 13-0112-85; dilution, 1:100), anti-B220-biotin (clone. no. RA3-6B2; eBioscience; cat. no. 13-0452-85; dilution, 1:100), anti-CD3e-biotin (clone no. 145-2C11; eBioscience; cat. no. 13-0031-85; dilution, 1:100), anti-Gr-1-biotin (clone. no. RB6-8C5; dilution, 1:100), anti-Ter119-biotin (clone no. TER-119; eBioscience; cat. no. 13-5921-85; eBioscience; cat. no. 13-5931-85; dilution, 1:100), anti-Sca-1-PE (clone no. D7; eBioscience; cat. no. 12-5981-83; dilution, 1:100) and anti-c-Kit-APC (clone no. 2B8; eBioscience; cat. no. 17-1171-83; dilution, 1:100) was added to the cell suspension $\left(1-2 \times 10^{6}\right.$ cells $\left./ 100 \mu \mathrm{l}\right)$ and incubated at room temperature in the dark for $15 \mathrm{~min}$. After washing the cells with 2\% FCS-PBS three times, the cells labelled with biotinylated antibodies were incubated with a streptavidin-FITC secondary antibody (eBioscience; cat. no. 11-4317-87; dilution, 1:100) at room temperature in the dark for $15 \mathrm{~min}$. The cells were subsequently washed three 
Table I. Inhibitor combinations and corresponding abbreviations.

\begin{tabular}{lc}
\hline Group & Abbreviation \\
\hline Freshly isolated & $\mathrm{A}$ \\
DMSO & $\mathrm{B}$ \\
VPA & $\mathrm{C}$ \\
SB203580 & $\mathrm{D}$ \\
Rapamycin & $\mathrm{E}$ \\
SB216763 & $\mathrm{F}$ \\
SB203580 + SB216763 & $\mathrm{G}$ \\
SB203580 + VPA & $\mathrm{H}$ \\
SB216763 + VPA & $\mathrm{I}$ \\
Rapamycin + SB216763 & $\mathrm{J}$ \\
SB203580 + rapamycin & $\mathrm{K}$ \\
Rapamycin + VPA & $\mathrm{L}$ \\
SB203580 + rapamycin + SB216763 & $\mathrm{M}$ \\
SB203580 + rapamycin + VPA & $\mathrm{N}$ \\
SB203580 + VPA + SB216763 & $\mathrm{O}$ \\
Rapamycin + SB216763 + VPA & $\mathrm{P}$ \\
VPA, valproic acid & sodium salt
\end{tabular}

$1 \mu \mathrm{M}$ for the P38MAPK signaling pathway inhibitor SB203580; $15 \mathrm{nM}$ for the mTOR signaling pathway inhibitor Rapamycin; $500 \mathrm{nM}$ for the GSK3 $\beta$ signaling pathway inhibitor CHIR99021; and $1 \mathrm{mM}$ for the HDAC signaling pathway inhibitor VPA. We now combine these inhibitors in different combinations and represent them with corresponding signaling pathway names, and finally we use the letters A-Q for each combination.

times with PBS and resuspended in 2\% FCS-PBS, and the LSK hematopoietic progenitor cells were subsequently sorted by flow cytometry on a Moflo XDP cell sorter.

The sorted cells were then cultured in DMEM (Sigma-Aldrich; Merck KGaA), supplemented with 10\% FBS (Gibco; Thermo Fisher Scientific), penicillin-streptomycin and gentamicin in humidified incubators with anti-vibration platform at $37^{\circ} \mathrm{C}$ with $5 \% \mathrm{CO}_{2}$. The medium also contained $20 \mathrm{ng} / \mathrm{ml}$ TPO (cat. no. 315-14-10), $20 \mathrm{ng} / \mathrm{ml} \mathrm{SCF}$ (cat. no. 250-03-50), $20 \mathrm{ng} / \mathrm{ml}$ Flt3-ligand (cat. no. 250-31L-10) (all from PeproTech, Inc.), $10 \mu \mathrm{g} / \mathrm{ml}$ heparin and $0.1 \mathrm{mM} \beta$-mercaptoethanol.

For treatment, the cells were treated with combinations of SB203580, CHIR99021, VPA, SB216763 and rapamycin at the indicated concentrations or DMSO (Ctr) at $37^{\circ} \mathrm{C}$ with $5 \% \mathrm{CO}_{2}$ for 9 days, followed by flow cytometric analysis.

The optimal inhibitory concentration of these five compounds had been obtained (SB216763, $1 \mu \mathrm{M}$; rapamycin, $15 \mathrm{nM}$; CHIR99021, $500 \mathrm{nM}$; VPA, $1 \mathrm{mM}$ ), cells were treated with different combinations of these inhibitors (Table I) at $37^{\circ} \mathrm{C}$ with $5 \% \mathrm{CO}_{2}$, then examined with under a Leica light microscope (magnification, $\mathrm{x} 40$ ).

Statistical analysis. Data are presented as the mean \pm SEM of three independent experiments. Statistical differences were determined using a one-way ANOVA, followed by Bonferroni correction, while the statistical differences between the groups presented in Fig. S6 were analyzed using an unpaired Student's t-test with GraphPad prism 6.02 (GraphPad Software, Inc.) software. $\mathrm{P}<0.05$ was considered to indicate a statistically significant difference.

\section{Results}

SB203580, an inhibitor of the p38MAPK signaling pathway, enhances in vitro HSC functionality. To determine how p38MAPK signaling influences HSC expansion in vitro, LSK cells were treated over a 9-day period using a specific inhibitor of this pathway, SB203580. The treatment with this inhibitor increased the number compared with the DMSO control (Fig. 1A and B). Significant changes were observed in the LSK cell counts in a dose-dependent manner. At 5-20 $\mu \mathrm{M}$, the number of cells began to decline, such that following the treatment with $1 \mu \mathrm{M} \mathrm{SB} 203580$, the number of LSK cells was significantly increased, while the frequency of Lin cells at this concentration did not significantly increase, compared with the DMSO control (Fig. 1C-G). In addition, at $1 \mu \mathrm{M}$, the absolute number of Lin cells (Fig. 1C) and the frequency and absolute of LSK (Fig. 1F and G) significantly increased, compared with DMSO. To improve on the accuracy of these results, whole bone marrow cells should be used for future experiments. These results suggested that inhibiting p38MAPK signaling may alter HSC differentiation and expansion in vitro.

Inhibition of GSK3 $\beta$ signaling significantly enhances HSC expansion in vitro. Given the complexities of the in vivo bone marrow microenvironment and the role of GSK3 $\beta$ as a regulator of HSC functionality (8), HSCs were treated with SB216763, a specific inhibitor of this pathway. At $2 \mu \mathrm{M}$, treatment with SB216763 led to changes in morphology and increased proliferation (Fig. 2A and B). In addition, an increase in the number of total cells (Fig. 2C), number of Lin cells (Fig. 2D), LSK cell proportion (Fig. 2E) and LSK cells absolute number (Fig. 2F) was also observed, compared with CHIR99021 treatment (Figs. 2 and S1). Although the increase amplitude of CHIR99021 was higher than that of SB216763 draft at $1 \mu \mathrm{M}$, the increase of LSK was not obvious at this concentration (Fig. 2C). By comparison, SB216763 was identified to more effectively enhance HSC proliferation, compared with CHIR99021 (Figs. 2, S2 and S3).

Based on these findings, it was hypothesized that the combined inhibition of p38MAPK and GSK3 $\beta$ signaling pathways may more effectively expand HSCs. Therefore, excluding the cytotoxic effect of DMSO on the cells (Fig. S4), the combination of SB203580 and SB216763 treatment was used to observe the expansion of HSCs; it was identified that the proportion of $\mathrm{Lin}^{-}$and LSK cells were not significantly different, compared with $1 \mu \mathrm{M}$ SB203580 treatment alone (Fig. S5). However, compared with the DMSO group, the total number of cells, the frequency and absolute of Lin- cells, the frequency and absolute of LSK cells of G group was significantly increased (Fig. S6 and Table I), suggesting that p38MAPK and GSK3 $\beta$ inhibitors may exert a synergistic effect in promoting HSCs expansion.

HDAC signaling inhibitor VPA alters HSC expansion in vitro. The highest total cell number (Fig. 3B), as well as relative and absolute Lin ${ }^{-}$and LSK cell numbers (Fig. 3C, E and G) 

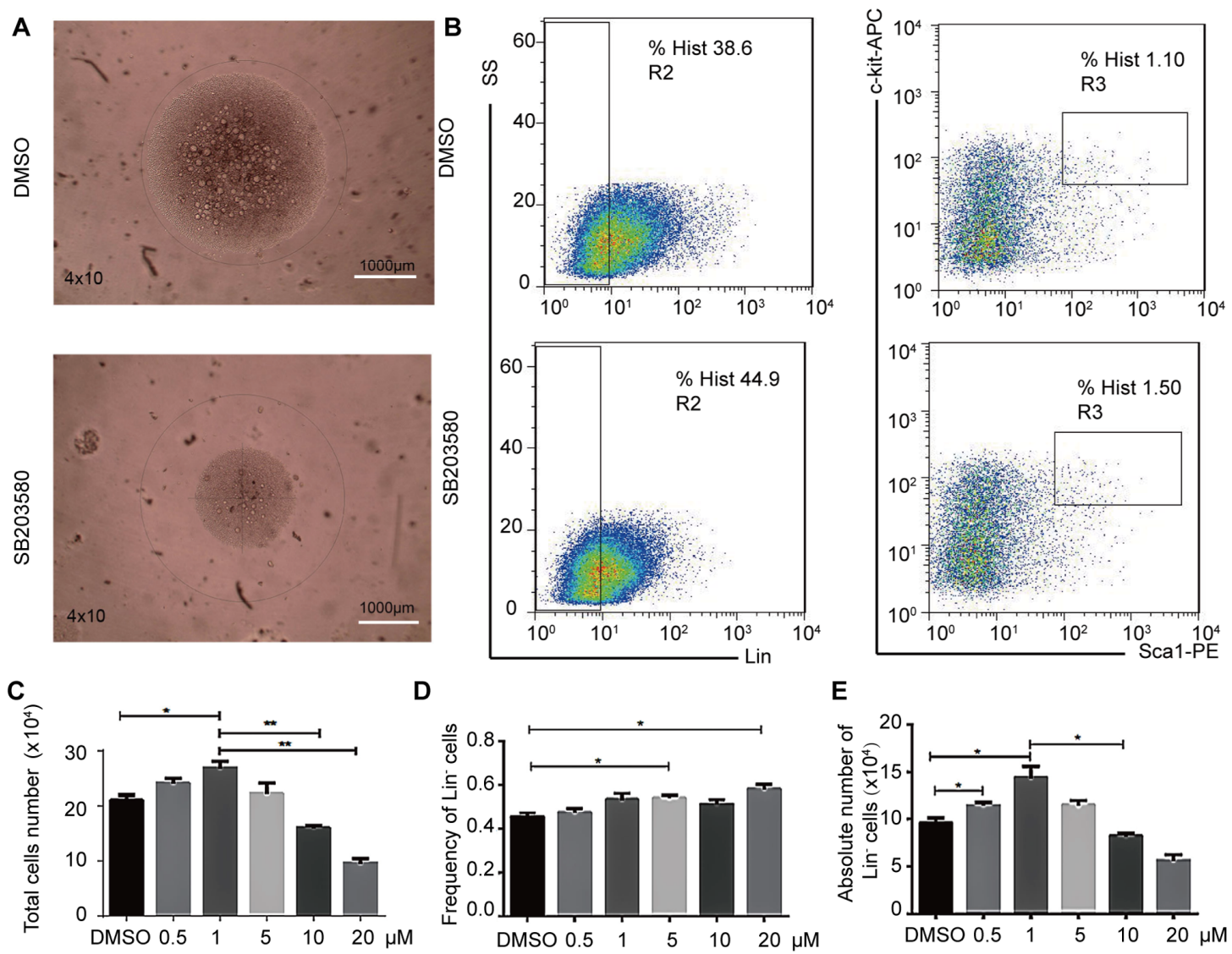

D

\section{E}
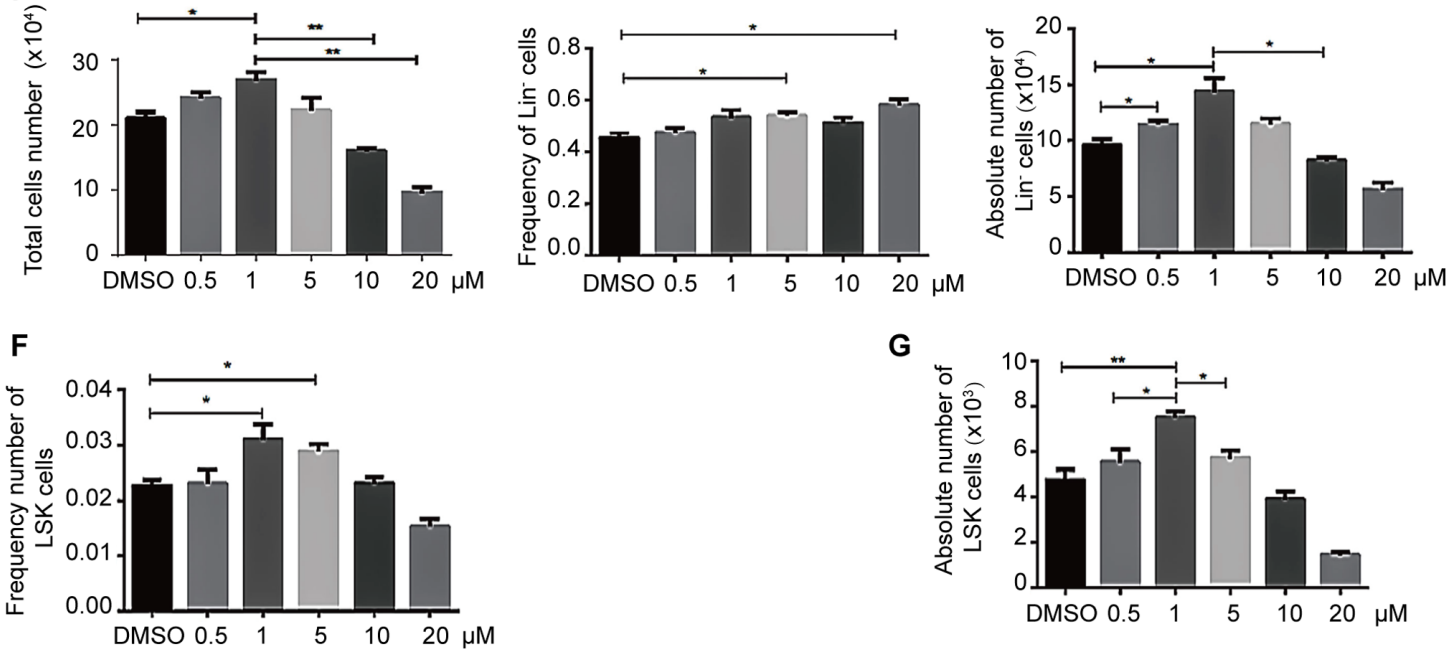

Figure 1. Effects of p38MAPK inhibition on hematopoietic stem cell expansion in vitro. LSK cells cultured in vitro for 9 days were first microscopically observed and photographed, and then were collected, stained with antibodies against Gr1, CD11b, Ter119, CD3e, B220, Sca-1, and c-Kit, then analyzed by flow cytometry. (A) LSK cell morphology. $\mathrm{n}=4$. Scale bar, 1,000- $\mu \mathrm{m}$. (B) Flow cytometric analysis of LSK cells treated with $1 \mu \mathrm{M}$ SB203580 or equal volume DMSO. $n=3$. (C) Total cell number. Magnification, $x 40$. Total images were obtained using the confocal Leica DM RXA microscope. (D) Relative and (E) absolute Lin cell numbers. (F) Relative and (G) absolute LSK cell numbers. $n=4 ;{ }^{*} \mathrm{P}<0.05,{ }^{* *} \mathrm{P}<0.01$. Lin, lineage; APC, allophycocyanin; PE, phycoerythrin; SS, side scatter; Sca-1, stem cell antigen-1.

were achieved at the $0.25 \mathrm{mM}$ dose of VPA (HDAC inhibitor). However, it was found that the frequency of Lin $^{-}$and LSK cells increased linearly with increasing VPA concentration (Fig. 3D and F). Meanwhile, when the VPA concentration was $1 \mathrm{mM}$, the number of cells was significantly reduced and were transparent and uniform, in a very good state under the microscope (Fig. 3A) and maintained the cells in an undifferentiated state. This $1 \mathrm{mM}$ dose was therefore used in the following experiments. At the same time, the number of Lin' cells was discovered to significantly increase in the $\mathrm{H}$ and I groups compared with $\mathrm{B}$, while the combination of VPA and other inhibitors exerted a minimal effect on stem cell expansion, demonstrating population frequencies similar to the DMSO control sample (Fig. S6 and Table I). Several groups of cells were seen to be highly differentiated, especially group $\mathrm{B}$. The number of differentiated cells was much higher than the other experimental groups, while groups $\mathrm{C}, \mathrm{H}, \mathrm{I}, \mathrm{N}, \mathrm{O}$ and $\mathrm{P}$ could maintain an even transparent morphology, but the cells barely grew after all four inhibitors were added, so we did not count this group when we collected the data, i.e., group $\mathrm{Q}$ above was invalid (Fig. S6 and Table I).

mTOR signaling inhibition alters in vitro HSC expansion. To determine how mTOR inhibition affects HSC functionality in vitro, cells were cultured with $(0,10,15,20,25$ or $30 \mathrm{nM})$ rapamycin for nine days, adding additional rapamycin $(0,10$, $15,20,25$ or $30 \mathrm{nM}$ ) every two days during culture to overcome metabolism of rapamycin. Following 9 days of rapamycin treatment, the cells were discovered to be healthy, with very few differentiated cells under the microscope, a lower number of cells overall and uniform cellular morphology (Fig. 4B), suggesting a rapamycin-dependent inhibition of cell growth. 
A
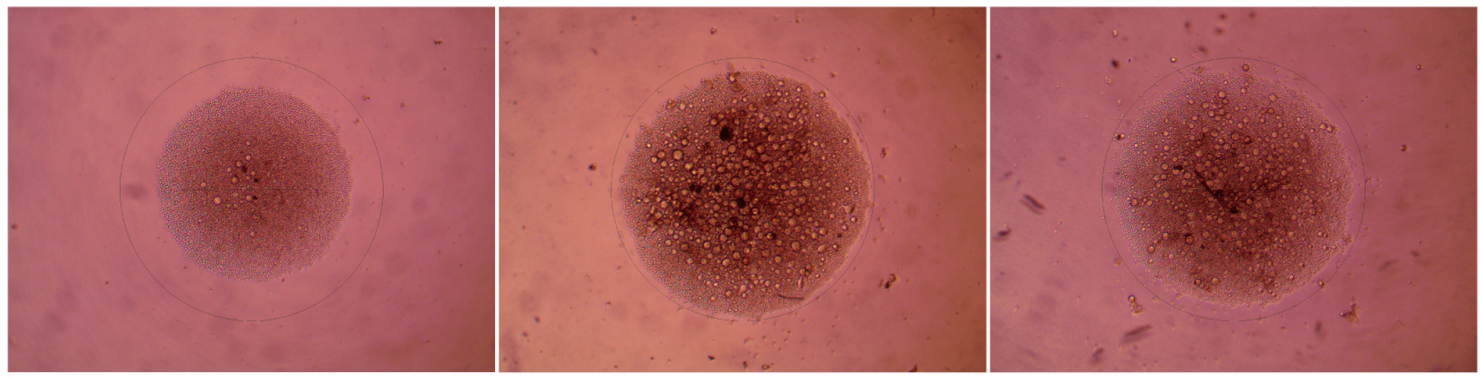

B

DMSO

CHIR99021

SB216763
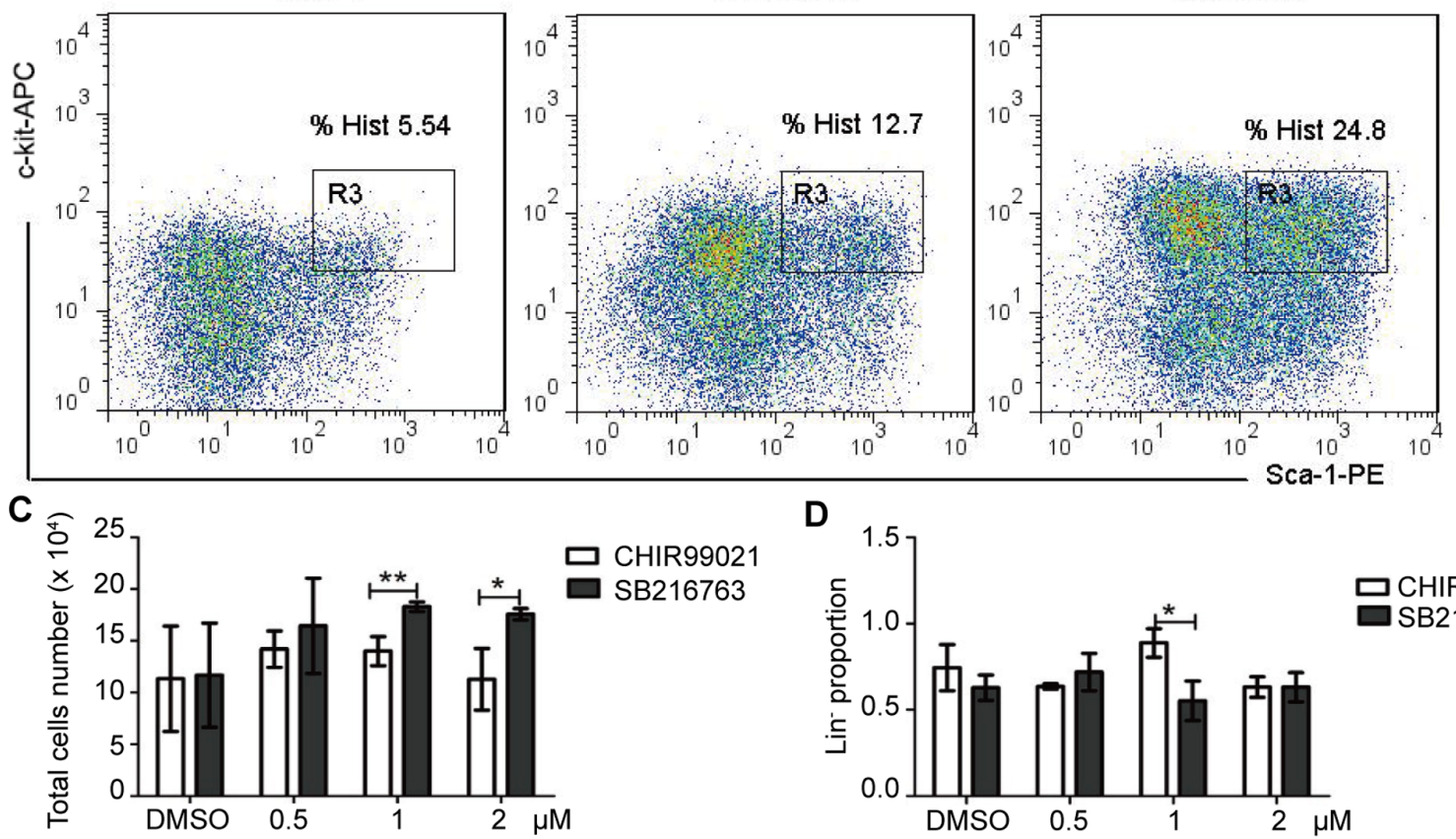

D

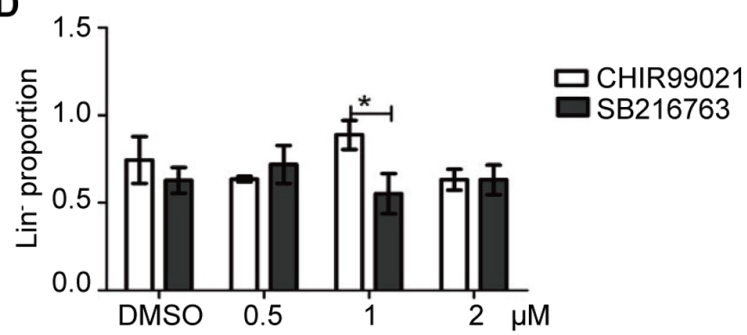

E
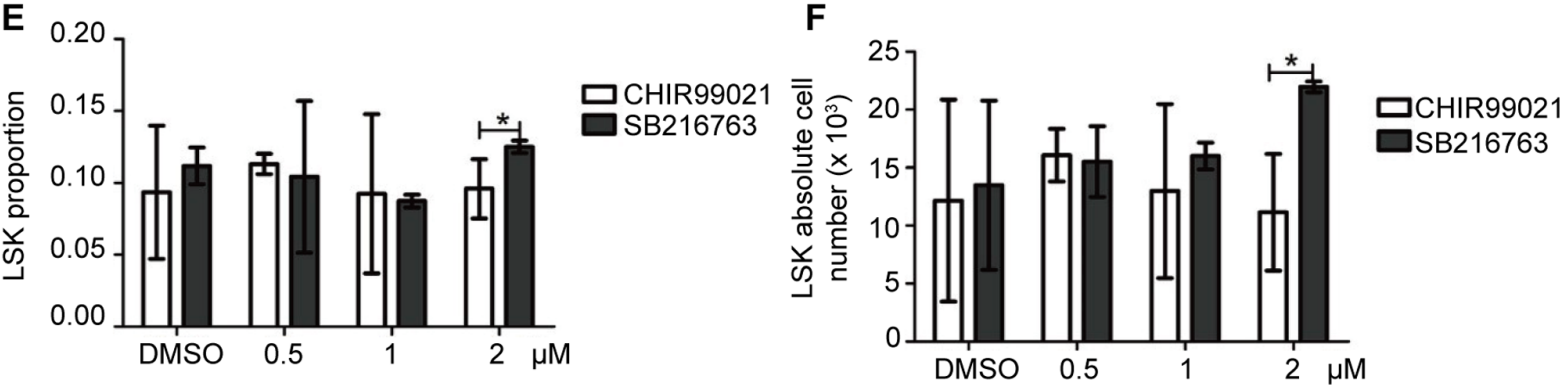

Figure 2. GSK3 $\beta$ inhibition alters hematopoietic stem cell expansion in vitro. The GSK3 $\beta$ inhibitors CHIR99021 $(0.5,1,2 \mu \mathrm{M})$ or SB216763 $(0.5,1,2 \mu \mathrm{M})$ were used to treat cells in media supplemented with cytokines for 9 days. (A) Cell morphology. Magnification, x40; scale bar, 1,000 $\mu \mathrm{m}$. Images were obtained using the confocal Leica DM RXA microscope. (B) After cultured in vitro for 9 days, cells were analyzed via flow cytometry to assess the percentage/number of LSK cells. (C) Total number of cell numbers following a 9-day culture. (D) Relative number of Lin cells. (E) Relative and (F) absolute LSK cell numbers. $\mathrm{n}=4 ;{ }^{*} \mathrm{P}<0.05,{ }^{* *} \mathrm{P}<0.01$. APC, allophycocyanin; PE, phycoerythrin; Lin, lineage; GSK3 $\beta$, glycogen synthase kinase 3 $\beta$; Sca-1, stem cell antigen-1.

The cells were further analyzed via flow cytometry, which revealed that in response to rapamycin treatment, there was a significant increase in the Lin ${ }^{-}$cell ratio (Fig. 4D and E), as well as in the frequency and absolute of LSK cells (Fig. 4F and G), while total cell numbers declined (Fig. 4C) which may be because rapamycin inhibits cell proliferation, thus maintaining cell stemness and inhibiting cell differentiation. Thus, $15 \mathrm{nM}$ was determined as a suitable inhibitory concentration for the mTOR signaling pathway inhibitor rapamycin. Similarly, the proportion of LSK cells in group K and $\mathrm{N}$ was increased compared with the other combinations in the random combination of inhibitors (Fig. S6 and Table I). These findings suggested that rapamycin may slow HSC growth, maintaining cells in a relatively undifferentiated and stem-like state.

\section{Discussion}

AML is a very common type of cancer; however, patients with AML often still have a poor prognosis (49). The majority of patients currently receive stem cell transplantation therapy as a means of improving patient outcomes and prolonging 

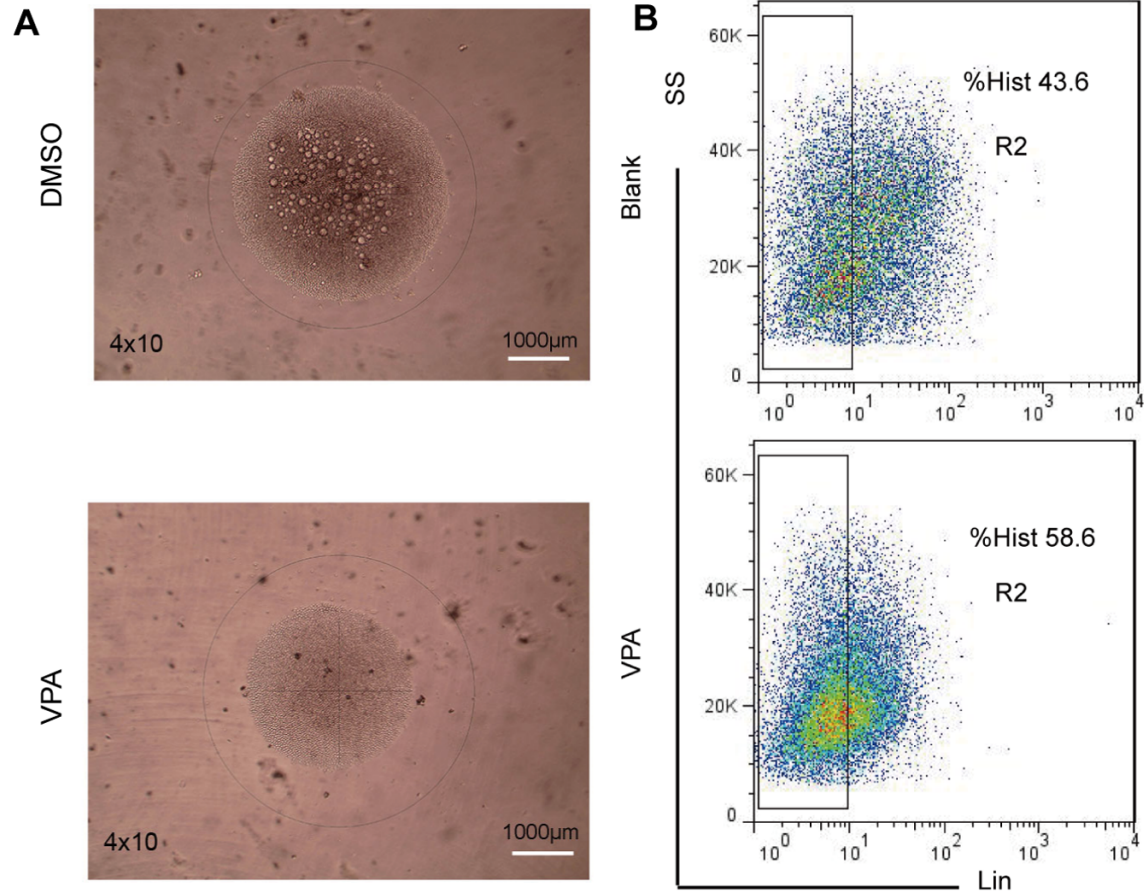

C

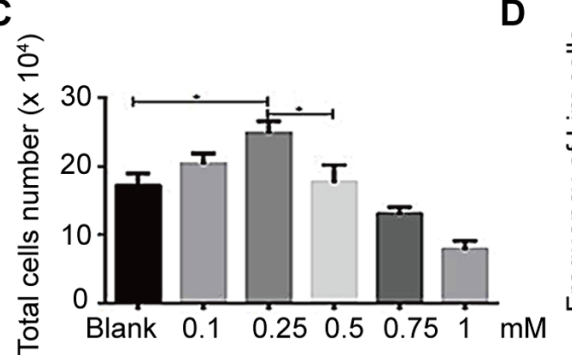

$\mathbf{F}$

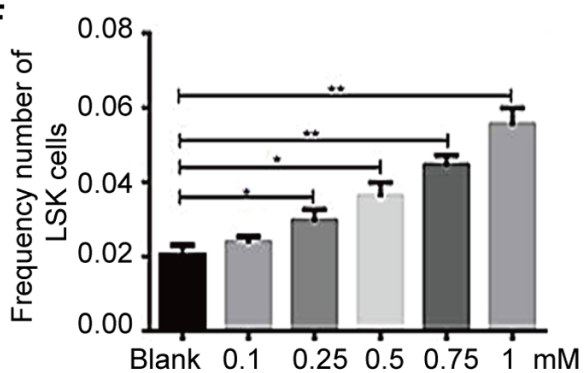

G
B
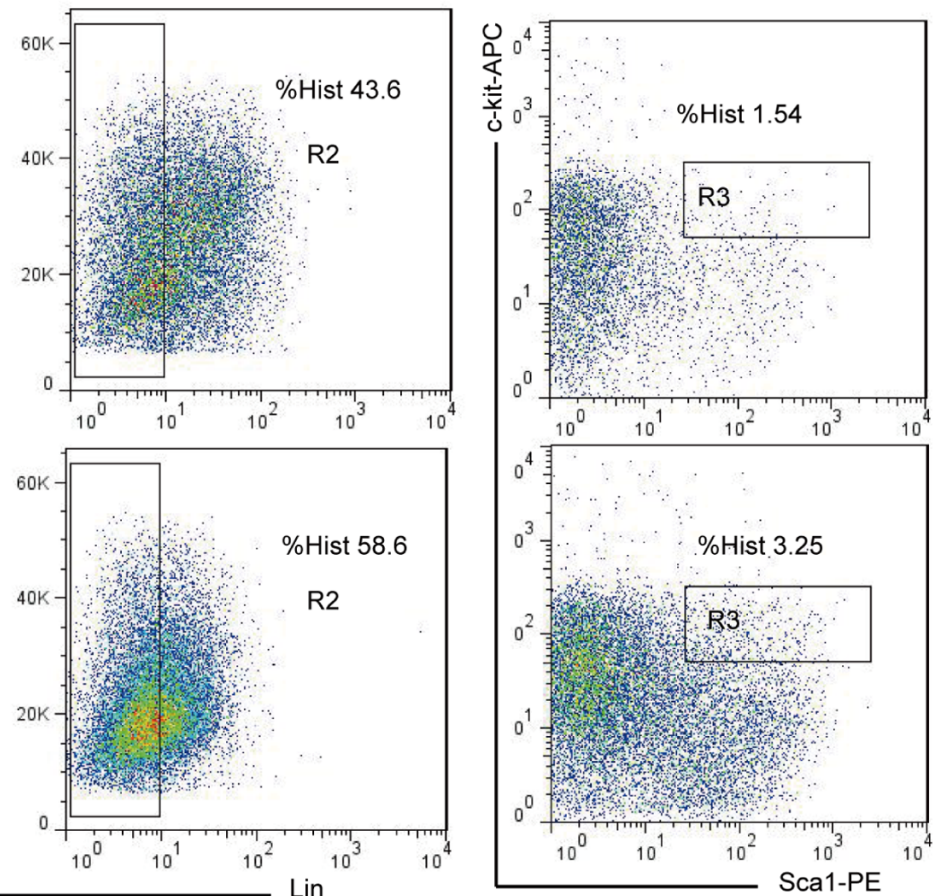

E

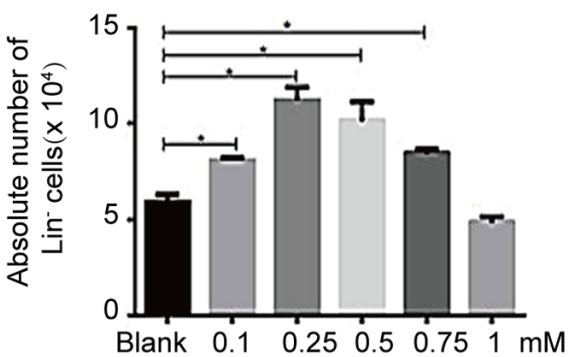

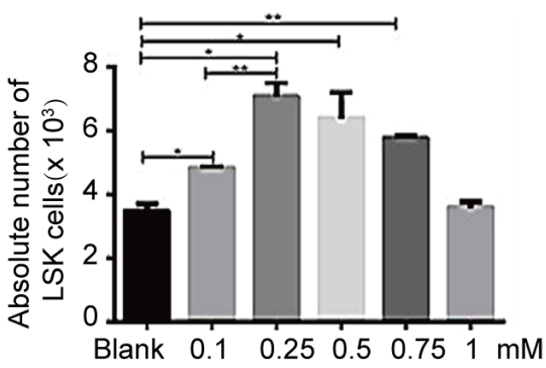

Figure 3. VPA affects hematopoietic stem cell expansion in vitro. LSK cells treated with various concentrations of VPA $(0.1,0.25,0.5,0.75,1 \mathrm{mM}) \mathrm{were}$ analyzed by flow cytometry after a 9-day culture period. Cells were stained for the expression of Gr1, CD11b, Ter119, CD3e, B220, Sca-1 and c-Kit. (A) LSK cell morphology was analyzed using a confocal Leica DM RXA microscope. Magnification, x40; scale bar, 1,000 $\mu \mathrm{m}$. (B) LSK cells were analyzed via flow cytometry. (C) Total cell number. (D) Lin cell frequency. (E) Lin cell number. (F) LSK cell frequency. (G) LSK cell number. $\mathrm{n}=4 ;{ }^{-}{ }^{-} \mathrm{P}<0.05,{ }^{* *} \mathrm{P}<0.01$, ${ }^{* * *} \mathrm{P}<0.001$. APC, allophycocyanin; PE, phycoerythrin; Lin, lineage; SS, side scatter; VPA, valproic acid sodium salt; Sca-1, stem cell antigen-1.

survival (50). For such HSC transplantation (HCT) procedures, donor stem cells are typically derived either from bone marrow, peripheral blood or umbilical cord blood (51). As the number of HSCs available from these donor tissues, and particularly in cord blood samples, can often be very limited, this can lead to very long recovery periods, making it a priority to identify novel means of enhancing HSC expansion in vitro (52-54). However, HSC differentiation and homeostatic regulation in vivo depends upon a wide array of complex microenvironmental inputs that can be difficult to replicate in vitro $(55,56)$.

While there have been numerous efforts made to date to promote ex vivo HSC expansion to improve engraftment rates in the clinical setting $(57,58)$, there still remains a significant unmet need in the field of HCT, and as such novel, ex vivo expansion strategies are still required. Numerous previous studies revealed that 338 MAPK, mTOR, GSK3 $\beta$ and HDAC signaling were all important for regulating the proliferation, differentiation, apoptosis and necrosis of HSCs (7,59-63). Therefore, the present study hypothesized that the simultaneous inhibition of multiple of these pathways may promote the synergistic expansion of HSCs in vitro, thereby representing a potentially viable strategy for improving HCT outcomes in patients with leukemia.

In the present study, the expansion of cells using a single molecule inhibitor revealed that the HSC expansion efficiency 

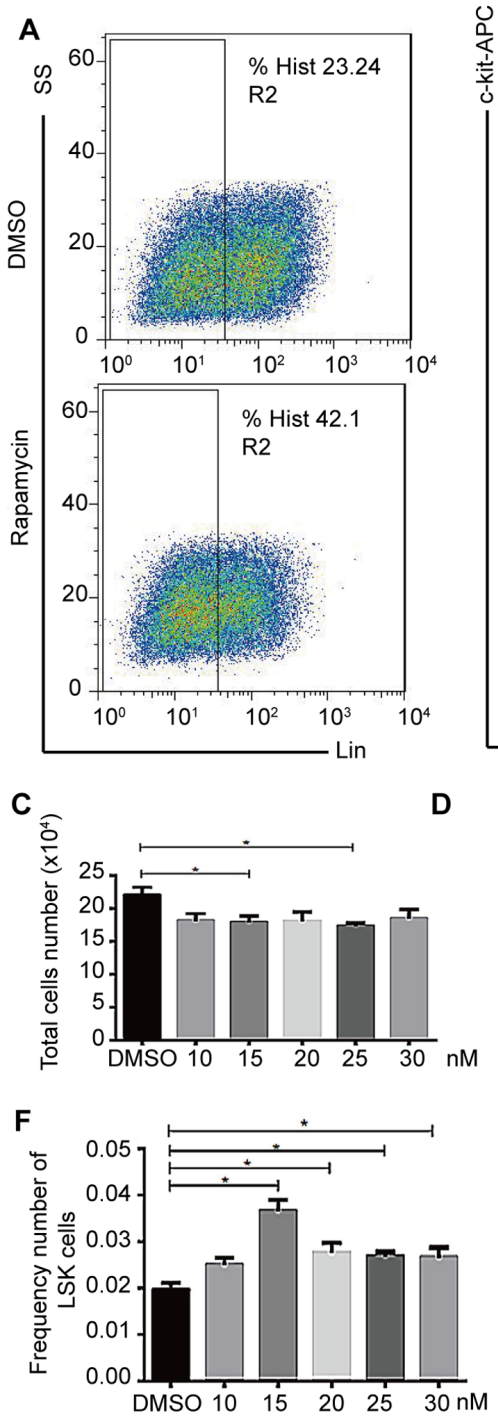

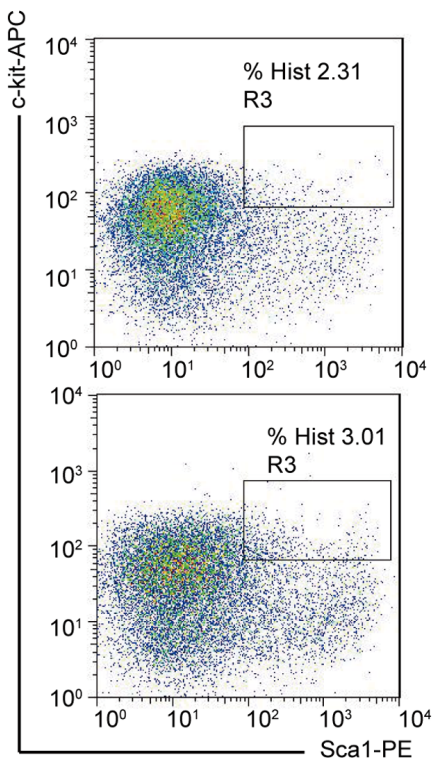

B
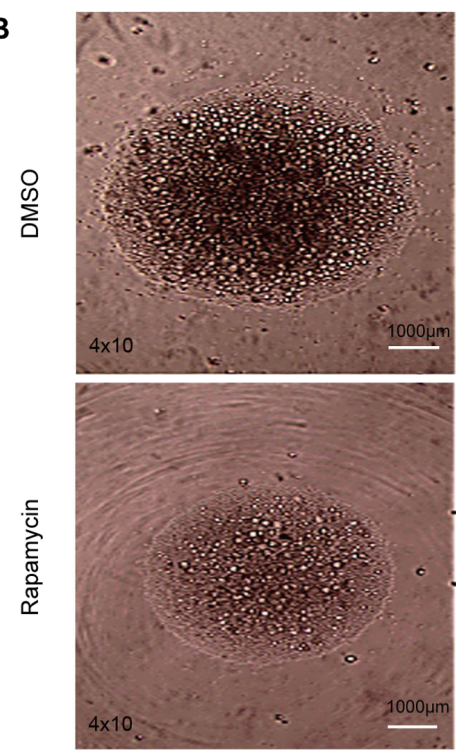

E
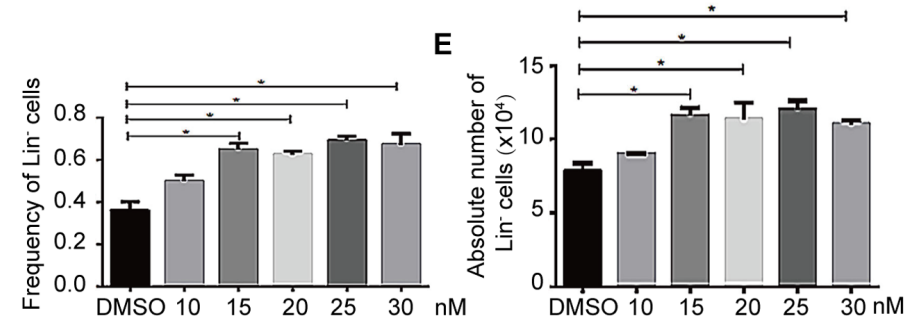

G

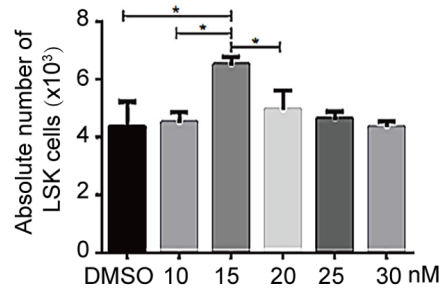

Figure 4. mTOR inhibition affects hematopoietic stem cell expansion in vitro. LSK cells were treated with rapamycin (10, 15, 20, 25 and $30 \mathrm{nM})$ or DMSO. (A) LSK cells were analyzed via flow cytometry. (B) Cell morphology following rapamycin or DMSO treatment. Magnification, $\mathrm{x} 40$; scale bar, 1,000- $\mu \mathrm{m}$. Cells were visualized using the confocal Leica DM RXA microscope. (C) Total cell number following rapamycin (10, 15, 20, 25 and $30 \mathrm{nM})$ or equal volume DMSO treatment. (D) Lin cell frequency following rapamycin or equal volume DMSO treatment. (E) Lin cell number following rapamycin or DMSO treatment. (F) LSK cell frequency following rapamycin $(10,15,20,25$ and $30 \mathrm{nM})$ or equal volume DMSO treatment. (G) LSK cell number following rapamycin (10, 15, 20,25 and $30 \mathrm{nM}$ ) or equal volume DMSO treatment. $\mathrm{n}=4$. ${ }^{*} \mathrm{P}<0.05$, APC, allophycocyanin; PE, phycoerythrin; Lin, lineage; SS, side scatter; Sca-1, stem cell antigen-1.

was significantly increased when SB203580 and SB216763 were used alone. HDAC signaling was also discovered to be a crucial regulator of HSC homeostasis (64). It was therefore investigated how the suppression of HDAC signaling may alter HSC functionality in an in vitro experimental system. High VPA concentrations failed to enhance HSC proliferation, which may have been potentially due to the cytotoxic effects of high concentrations of this inhibitor, which induced apoptotic signaling, and HDAC inhibition was sufficient to maintain HSCs in an undifferentiated state in vitro, enhancing their expansion $(65,66)$. Our studies demonstrated that HDAC inhibitors induced HSC expansion, maintained undifferentiated cells in vitro. mTOR signaling regulates the growth and metabolism of cells, in addition to regulating HSC homing $(62,67,68)$. mTOR activation has also been illustrated to disrupt HSC quiescence and drive HSC exhaustion, whereas inhibiting this signaling pathway restored
HSC self-renewal $(61,69)$. In the present study, inhibiting the mTOR pathway led to decreased cell growth, but also helped maintain the cells in a more stem-like undifferentiated state. These results suggested that the mTOR signaling pathway may serve an important role in regulating the self-renewal of HSCs in vitro. Thus, inhibiting the mTOR pathway using rapamycin may represent a novel approach to promote HSC expansion in vitro to improve HCT outcomes.

Next, to investigate how combinations of these signaling pathway inhibitors affect HSC expansion and differentiation in vitro, cells were treated with combinations of the optimal concentrations of these pathway-specific inhibitors. Consistent with our hypothesis, it was discovered that the combination of SB203580 and SB216763 significantly increased HSC differentiation in vivo compared with the other treatment groups. The combined inhibition of p38MAPK and mTOR was also able to amplify HSCs in vitro, although not as effectively 
as the combination of p38MAPK and GSK-3 $\beta$ inhibition. In contrast, the treatment of cells with HDAC inhibitors was associated with an almost complete loss of differentiation (70), suggesting that HDAC inhibitors may damage cellular responses in this context. Among all the important signaling pathways, using these various combinations, the results indicated that through combining the p38MAPK and GSK $3 \beta$ inhibitors, HSC expansion was significantly enhanced in vitro.

In conclusion, the findings of the present study may provide a novel approach to expand HSCs ex vivo via inhibiting p38MAPK and GSK3 $\beta$, either alone or in combination. However, the specific molecular mechanisms through which p38MAPK and GSK3 $\beta$ signaling altered quiescent HSC expansion and proliferation remain unclear and warrant further investigations. Taken together, the aim of future studies is to obtain a series of small-molecule compounds that inhibit p38MAPK and GSK $3 \beta$ signaling pathways that can expand human HSCs ex vivo.

\section{Acknowledgements}

The authors thank the staff of the Department of Biology, College of Life and Sciences Shanghai Normal University for animal care services.

\section{Funding}

The present study was supported by the Leukemia Research Foundation New Investigator Award to JL (grant no. 81670151) and the National Natural Science Foundation of China (grant no. 81700141).

\section{Availability of data and materials}

The datasets used and/or analyzed during the current study are available from the corresponding author on reasonable request.

\section{Authors' contributions}

YJ performed the experiments, analyzed and extracted the data, generated the figures and wrote the manuscript. ZX, $\mathrm{NM}$ and LY analyzed the data; $\mathrm{CH}$ conceived the project and reviewed the paper. JL conceived the project, designed and performed the experiments, and analyzed and interpreted results. All authors read and approved the final manuscript.

\section{Ethics approval and consent to participate}

All animal studies were approved by the Institutional Animal Care and Use Committee of Shanghai Normal University.

\section{Patient consent for publication}

Not applicable.

\section{Competing interests}

The authors declare that they have no competing interests.

\section{References}

1. Shlush LI, Zandi S, Mitchell A, Chen WC, Brandwein JM, Gupta V, Kennedy JA, Schimmer AD, Schuh AC, Yee KW, et al: Identification of pre-leukaemic haematopoietic stem cells in acute leukaemia. Nature 506: 328-333, 2014.

2. Tijaro-Ovalle NM, Karantanos T, Wang HT and Boussiotis VA: Metabolic targets for improvement of allogeneic hematopoietic stem cell transplantation and graft-vs.-host disease. Front Immunol 10: 295, 2019.

3. Cui P, Zhang Y, Cui M, Li Z, Ma G, Wang R, Wang N, Huang S and Gao J: Leukemia cells impair normal hematopoiesis and induce functionally loss of hematopoietic stem cells through immune cells and inflammation. Leuk Res 65: 49-54, 2018.

4. Clements WK and Traver D: Signalling pathways that control vertebrate haematopoietic stem cell specification. Nat Rev Immunol 13: 336-348, 2013.

5. Harada K, Fuji S, Seo S, Kanda J, Ueki T, Kimura F, Kato K, Uchida N, Ikegame K, Onizuka M, et al: Comparison of the outcomes after haploidentical and cord blood salvage transplantations for graft failure following allogeneic hematopoietic stem cell transplantation. Bone Marrow Transplant 55: 1784-1795, 2020.

6. Ozdemir ZN and Civriz Bozdag S: Graft failure after allogeneic hematopoietic stem cell transplantation. Transfus Apher Sci 57: 163-167, 2018.

7. Karigane D, Kobayashi H, Morikawa T, Ootomo Y, Sakai M, Nagamatsu G, Kubota Y, Goda N, Matsumoto M, Nishimura EK, et al: $\mathrm{p} 38 \alpha$ activates purine metabolism to initiate hematopoietic stem/progenitor cell cycling in response to stress. Cell stem cell 19: 192-204, 2016.

8. Guezguez B, Almakadi M, Benoit YD, Shapovalova Z, Rahmig S, Fiebig-Comyn A, Casado FL, Tanasijevic B, Bresolin S, Masetti R, et al: GSK3 deficiencies in hematopoietic stem cells initiate pre-neoplastic state that is predictive of clinical outcomes of human acute leukemia. Cancer Cell 29: 61-74, 2016.

9. Zhou F, Li X, Wang W, Zhu P, Zhou J, He W, Ding M, Xiong F, Zheng X, Li Z, et al: Tracing haematopoietic stem cell formation at single-cell resolution. Nature 533: 487-492, 2016.

10. Hinge A, Xu J, Javier J, Mose E, Kumar S, Kapur R, Srour EF, Malik P, Aronow BJ and Filippi MD: p190-B RhoGAP and intracellular cytokine signals balance hematopoietic stem and progenitor cell self-renewal and differentiation. Nat Commun 8: $14382,2017$.

11. Kwon B: p38 $\alpha$-mediated purine metabolism is linked to exit from quiescence of hematopoietic stem cells. Stem Cell Investig 3: 69, 2016.

12. Ito K, Hirao A, Arai F, Takubo K, Matsuoka S, Miyamoto K, Ohmura M, Naka K, Hosokawa K, Ikeda Y and Suda T: Reactive oxygen species act through p38 MAPK to limit the lifespan of hematopoietic stem cells. Nat Med 12: 446-451, 2006.

13. Ratajczak MZ and Suszynska M: Emerging strategies to enhance homing and engraftment of hematopoietic stem cells. Stem Cell Rev Rep 12: 121-128, 2016.

14. Abdelbaset-Ismail A, Borkowska-Rzeszotek S, Kubis E, Bujko K, Brzeźniakiewicz-Janus K, Bolkun L, Kloczko J, Moniuszko M, Basak GW, Wiktor-Jedrzejczak W and Ratajczak MZ: Activation of the complement cascade enhances motility of leukemic cells by downregulating expression of heme oxygenase 1 (HO-1). Leukemia 31: 446-458, 2016.

15. Gao F and Liu WJ: Advance in the study on p38 MAPK mediated drug resistance in leukemia. Eur Rev Med Pharmacol Sci 20: 1064-1070, 2016.

16. Ko KH, Holmes T, Palladinetti P, Song E, Nordon R, O'Brien TA and Dolnikov A: GSK-3 $\beta$ inhibition promotes engraftment of ex vivo-expanded hematopoietic stem cells and modulates gene expression. Stem Cells 29: 108-118, 2011.

17. Huang J, Zhang Y, Bersenev A, O'Brien WT, Tong W, Emerson SG and Klein PS: Pivotal role for glycogen synthase kinase-3 in hematopoietic stem cell homeostasis in mice. J Clin Invest 119: 3519-3529, 2009.

18. Li H, Feng J, Zhang Y, Feng J, Wang Q, Zhao S, Meng P and Li J: Mst1 deletion attenuates renal ischaemia-reperfusion injury: The role of microtubule cytoskeleton dynamics, mitochondrial fission and the GSK3 $\beta$-p53 signalling pathway. Redox Biol 20: 261-274, 2019.

19. Lechman ER, Gentner B, van Galen P, Giustacchini A, Saini M, Boccalatte FE, Hiramatsu H, Restuccia U, Bachi A, Voisin V, et al: Attenuation of miR-126 activity expands HSC in vivo without exhaustion. Cell Stem Cell 11: 799-811, 2012. 
20. Lapid K, Itkin T, D'Uva G, Ovadya Y, Ludin A, Caglio G, Kalinkovich A, Golan K, Porat Z, Zollo M and Lapidot T: GSK3 $\beta$ regulates physiological migration of stem/progenitor cells via cytoskeletal rearrangement. J Clin Invest 123: $1705-1717,2013$

21. Gatla HR, Muniraj N, Thevkar P, Yavvari S, Sukhavasi S and Makena MR: Regulation of chemokines and cytokines by histone deacetylases and an update on histone decetylase inhibitors in human diseases. Int J Mol Sci 20: 1110, 2019.

22. Stahl M, Gore SD, Vey N and Prebet T: Lost in translation? Ten years of development of histone deacetylase inhibitors in acute myeloid leukemia and myelodysplastic syndromes. Expert Opin Investig Drugs 25: 307-317, 2016.

23. Jabbour E and Garcia-Manero G: Deacetylase inhibitors for the treatment of myelodysplastic syndromes. Leuk Lymphoma 56 1205-1212, 2015.

24. Liu Z, Ding K, Li L, Liu H, Wang Y, Liu C and Fu R: A novel histone deacetylase inhibitor Chidamide induces G0/G1 arrest and apoptosis in myelodysplastic syndromes. Biomed Pharmacother 83: 1032-1037, 2016.

25. Heuser M, Yun $\mathrm{H}$ and Thol F: Epigenetics in myelodysplastic syndromes. Semin Cancer Biol 51: 170-179, 2018.

26. Mao J, Li S, Zhao H, Zhu Y, Hong M, Zhu H, Qian S and Li J: Effects of chidamide and its combination with decitabine on proliferation and apoptosis of leukemia cell lines. Am J Transl Res 10: 2567-2578, 2018

27. Qi J, Singh S, Hua WK, Cai Q, Chao SW, Li L, Liu H, Ho Y, McDonald T, Lin A, et al: HDAC8 inhibition specifically targets Inv (16) acute myeloid leukemic stem cells by restoring p53 acetylation. Cell Stem Cell 17: 597-610, 2015

28. Chen C, Liu Y, Liu Y and Zheng P: mTOR regulation and therapeutic rejuvenation of aging hematopoietic stem cells. Sci Signal 2: ra75, 2009.

29. Cao D, Song J, Ling S, Niu S, Lu L, Cui Z, Li Y, Hao S, Zhong G, Qi Z, et al: Hematopoietic stem cells and lineage cells undergo dynamic alterations under microgravity and recovery conditions. FASEB J 33: 6904-6918, 2019.

30. Kassim AA and Savani BN: Hematopoietic stem cell transplantation for acute myeloid leukemia: A review. Hematol Oncol Stem Cell Ther 10: 245-251, 2017.

31. Chhabra A, Ring AM, Weiskopf K, Schnorr PJ, Gordon S, Le AC, Kwon HS, Ring NG, Volkmer J, Ho PY, et al: Hematopoietic stem cell transplantation in immunocompeten hosts without radiation or chemotherapy. Sci Transl Med 8: 351ra105, 2016.

32. De Meyts P: The Insulin Receptor and Its Signal Transduction Network. In: Endotext. Feingold KR, Anawalt B Boyce A, et al (eds). MDText.com, Inc., South Dartmouth, MA, 2000.

33. Bari S, Zhong Q, Fan X, Poon Z, Lim AST, Lim TH, Dighe N, Li S, Chiu GNC, Chai CLL and Hwang WYK: Ex vivo expansion of $\mathrm{CD} 34^{+} \mathrm{CD} 90^{+} \mathrm{CD} 49 \mathrm{f}^{+}$hematopoietic stem and progenitor cells from non-enriched umbilical cord blood with azole compounds. Stem Cells Transl Med 7: 376-393, 2018.

34. Cao R, Li L, Ying Z, Cao Z, Ma Y, Mao X, Li J, Qi X, Zhang Z and Wang X: A small molecule protects mitochondrial integrity by inhibiting mTOR activity. Proc Natl Acad Sci USA 116 23332-23338, 2019.

35. Dong G, Chen W, Wang X, Yang X, Xu T, Wang P, Zhang W, Rao Y, Miao C and Sheng C: Small molecule inhibitors simultaneously targeting cancer metabolism and epigenetics: Discovery of novel nicotinamide phosphoribosyltransferase (NAMPT) and histone deacetylase (HDAC) dual inhibitors. J Med Chem 60 : 7965-7983, 2017

36. Yu VW and Scadden DT: Hematopoietic stem cell and its bone marrow niche. Curr Top Dev Biol 118: 21-44, 2016.

37. Wilkinson AC, Ishida R, Kikuchi M, Sudo K, Morita M, Crisostomo RV, Yamamoto R, Loh KM, Nakamura Y, Watanabe M, et al: Long-term ex vivo haematopoietic-stem-cell expansion allows nonconditioned transplantation. Nature 571: $117-121,2019$.

38. Höfer T and Rodewald HR: Differentiation-based model of hematopoietic stem cell functions and lineage pathways. Blood 132: $1106-1113,2018$

39. Xu ZY, Chai S, Zhang XQ, Cao Y, Lian CQ, Wu WJ and Li YY: Influence of BMP4 on regulation of cell cycle and apoptosis of hematopoietic stem cells/progenitor cells and its mechanism in chemotherapy-induced myelosuppression. Zhongguo Shi Yan Xue Ye Xue Za Zhi 27: 1265-1271, 2019 (in Chinese).
40. Morcos MNF, Schoedel KB, Hoppe A, Behrendt R, Basak O, Clevers HC, Roers A and Gerbaulet A: SCA-1 expression level identifies quiescent hematopoietic stem and progenitor cells. Stem Cell Rep 8: 1472-1478, 2017.

41. Wang Y, Kellner J, Liu L and Zhou D: Inhibition of p38 mitogen-activated protein kinase promotes ex vivo hematopoietic stem cell expansion. Stem Cells Dev 20: 1143-1152, 2011.

42. Cirillo PF, Pargellis C and Regan J: The non-diaryl heterocycle classes of p38 MAP kinase inhibitors. Curr Top Med Chem 2: $1021-1035,2002$

43. Gao LY, Zhao MY, Li P, Kong J, Liu Z, Chen Y, Huang R, Chu J, Quan J and Zeng R: Glycogen synthase kinase 3 (GSK3)-inhibitor SB216763 promotes the conversion of human umbilical cord mesenchymal stem cells into neural precursors in adherent culture. Hum Cell 30: 11-22, 2017

44. Zeng J, Liu X, Li X, Zheng Y, Liu B and Xiao Y: Daucosterol inhibits the proliferation, migration, and invasion of hepatocellular carcinoma cells via Wnt//3-catenin signaling. Molecules 22: 862,2017

45. Cheng T, Zhai K, Chang Y, Yao G, He J, Wang F, Kong H, Xin H, Wang H, Jin M, et al: CHIR99021 combined with retinoic acid promotes the differentiation of primordial germ cells from human embryonic stem cells. Oncotarget 8: 7814-7826, 2017.

46. Dobzanski A, Khalil SM and Lane AP: Nasal polyp fibroblasts modulate epithelial characteristics via Wnt signaling. Int Forum Allergy Rhinol 8: 1412-1420, 2018.

47. Wu Y, Ai Z, Yao K, Cao L, Du J, Shi X, Guo Z and Zhang Y: CHIR99021 promotes self-renewal of mouse embryonic stem cells by modulation of protein-encoding gene and long intergenic non-coding RNA expression. Exp Cell Res 319: 2684-2699, 2013

48. Mahajan MM, Cheng B, Beyer AI, Mulvaney US, Wilkinson MB, Fomin ME and Muench MO: A quantitative assessment of the content of hematopoietic stem cells in mouse and human endosteal-bone marrow: A simple and rapid method for the isolation of mouse central bone marrow. BMC Hematol 15: 9, 2015.

49. DiNardo CD and Cortes JE: Mutations in AML: Prognostic and therapeutic implications. Hematology Am Soc Hematol Educ Program 2016: 348-355, 2016.

50. Cornelissen JJ and Blaise D: Hematopoietic stem cell transplantation for patients with AML in first complete remission. Blood 127: 62-70, 2016

51. Richter J, Traver D and Willert K: The role of Wnt signaling in hematopoietic stem cell development. Crit Rev Biochem Mol Biol 52: 414-424, 2017.

52. de Jamblinne Y, Baudoux E, Delo C and Coppieters Y: Influence of obstetric factors on characteristics of umbilical cord blood transplants. Gynecol Obstet Fertil Senol 46: 639-644, 2018 (In French).

53. Lou X, Zhao C and Chen H: Unrelated donor umbilical cord blood transplant versus unrelated hematopoietic stem cell transplant in patients with acute leukemia: A meta-analysis and systematic review. Blood Rev 32: 192-202, 2018.

54. Tong J, Xuan L, Sun Y, Huang D, Liu H, Zheng C, Zhu X, Tang B, Song K, Zhang X, et al: Umbilical cord blood transplantation without antithymocyte globulin results in similar survival but better quality of life compared with unrelated peripheral blood stem cell transplantation for the treatment of acute leukemia-a retrospective study in China. Biol Blood Marrow Transplant 23: 1541-1548, 2017.

55. Kaushansky $\mathrm{K}$ and $\mathrm{Zhan} \mathrm{H}$ : The regulation of normal and neoplastic hematopoiesis is dependent on microenvironmental cells. Adv Biol Regul 69: 11-15, 2018.

56. Lucas D: The bone marrow microenvironment for hematopoietic stem cells. Adv Exp Med Biol 1041: 5-18, 2017.

57. Dzierzak E and Bigas A: Blood development: Hematopoietic stem cell dependence and independence. Cell Stem Cell 22: 639-651, 2018.

58. Derakhshani M, Abbaszadeh $\mathrm{H}$, Movassaghpour A A, Mehdizadeh A, Ebrahimi-Warkiani M and Yousefi M: Strategies for elevating hematopoietic stem cells expansion and engraftment capacity. Life Sci 232: 116598, 2019.

59. Hua Y, Wang C, Jiang H, Wang Y, Liu C, Li L, Liu H, Shao Z and $\mathrm{Fu} \mathrm{R}$ : Iron overload may promote alteration of NK cells and hematopoietic stem/progenitor cells by JNK and p38 pathway in myelodysplastic syndromes. Int J Hematol 106: 248-257, 2017.

60. Tesio M, Tang Y, Mudder K, Saini M, von Paleske L, Macintyre E, Pasparakis M, Waisman A and Trumpp A: Hematopoietic stem cell quiescence and function are controlled by the CYLD-TRAF2-p38MAPK pathway. J Exp Med 212: 525-538, 2015. 
61. Peng H, Kasada A, Ueno M, Hoshii T, Tadokoro Y, Nomura N Ito C, Takase Y, Vu HT, Kobayashi M, et al: Distinct roles of Rheb and Raptor in activating mTOR complex 1 for the self-renewal of hematopoietic stem cells. Biochem Biophys Res Commun 495: 1129-1135, 2018.

62. Oburoglu L, Romano M, Taylor N and Kinet S: Metabolic regulation of hematopoietic stem cell commitment and erythroid differentiation. Curr Opin Hematol 23: 198-205, 2016.

63. Trowbridge JJ, Xenocostas A, Moon RT and Bhatia M: Glycogen synthase kinase-3 is an in vivo regulator of hematopoietic stem cell repopulation. Nature Med 12: 89-98, 2006.

64. Dhoke NR, Kalabathula E, Kaushik K, Geesala R, Sravani B and Das A: Histone deacetylases differentially regulate the proliferative phenotype of mouse bone marrow stromal and hematopoietic stem/progenitor cells. Stem Cell Res 17: 170-180, 2016.

65. Papa L, Djedaini M and Hoffman R: Ex vivo expansion of hematopoietic stem cells from human umbilical cord blood-derived CD34 ${ }^{+}$cells using valproic acid. J Vis Exp, 2019.

66. Aztopal N, Erkisa M, Erturk E, Ulukaya E, Tokullugil AH and Ari F: Valproic acid, a histone deacetylase inhibitor, induces apoptosis in breast cancer stem cells. Chem Biol Interact 280 51-58, 2018.
67. Zhang M, Liu F, Zhou P, Wang Q, Xu C, Li Y, Bian L, Liu Y, Zhou J, Wang F, et al: The mTOR signaling pathway regulates macrophage differentiation from mouse myeloid progenitors by inhibiting autophagy. Autophagy 15: 1150-1162, 2019.

68. Mossmann D, Park S and Hall MN: mTOR signalling and cellular metabolism are mutual determinants in cancer. Nat Rev Cancer 18: 744-757, 2018.

69. Luo Y, Li L, Zou P, Wang J, Shao L, Zhou D and Liu L: Rapamycin enhances long-term hematopoietic reconstitution of ex vivo expanded mouse hematopoietic stem cells by inhibiting senescence. Transplantation 97: 20-29, 2014.

70. Anastas JN, Zee BM, Kalin JH, Kim M, Guo R, Alexandrescu S, Blanco MA, Giera S, Gillespie SM, Das J, et al: Re-programing chromatin with a bifunctional LSD1/HDAC inhibitor induces therapeutic differentiation in DIPG. Cancer Cell 36: 528-544.e10, 2019.

This work is licensed under a Creative Commons Attribution-NonCommercial-NoDerivatives 4.0 International (CC BY-NC-ND 4.0) License. 